\title{
Fatigue life of cold-forging dies with various values of hardness
}

\author{
Yi-Che Lee, Fuh-Kuo Chen ${ }^{*}$ \\ Department of Mechanical Engineering, National Taiwan University, Section 4, No. 1, Roosevelt Road, Taipei 10764, Taiwan, ROC
}

\begin{abstract}
Four selected die materials commonly used in the cold-forging process were examined in the present study to obtain the relationship between the hardness and the die fatigue life. The die materials were first heat-treated by a developed process to obtain different values of hardness, while the ductility was retained at a favorable level. The material properties of these die materials were then obtained from tension and impact tests. By investigating the experimental data, the relationship between the mechanical properties and the hardness was established. A theoretical model was also proposed in the present study to predict the die fatigue life, considering the mechanical properties of the die material obtained in the experiments and the fatigue total strain as parameters. Since the material properties can be expressed in terms of hardness, it is convenient for a die design engineer to establish a simple guideline for the prediction of the fatigue life of coldforging dies using the proposed theoretical model. (C) 2001 Elsevier Science B.V. All rights reserved.
\end{abstract}

Keywords: Fatigue life; Cold-forging die; Hardness; Ductility; Theoretical model

\section{Introduction}

Since dies used in the cold-forging processes have to undergo severe impact and loading conditions, they are more prone to sudden fracture than most of the other machine components. To obtain an acceptable die life against fatigue failure, the ductility and hardness of the die material are the important factors in the production of the die. Usually, die materials must be hardened sufficiently to withstand severe service conditions, but also need to have enough ductility to prevent their cracking and brittle fracture, i.e. dies are designed for higher degrees of both hardness and ductility. However, a die with a higher degree of hardness could be associated with insufficient ductility due to a poor heattreatment process having been applied. In general, sufficient ductility of dies can be obtained by a proper heat-treatment process, but the hardness may vary over a wide range. Therefore, a heat-treatment process that produces a higher degree of hardness for the die material without loss of ductility is to be developed for actual practice, so that the die design engineer can measure only the hardness of the die material to estimate the die quality, assuming that the ductility of the die material is good to the specification. Following this concept, a simple guideline that associates the die fatigue life with the die hardness will be useful to engineers for die design.

\footnotetext{
${ }^{*}$ Corresponding author. Tel.: +886-2-3621-522; fax: +886-2-3631-755.
} E-mail address: fkchen@w3.me.ntu.edu.tw (F.-K. Chen).
In the present study, a heat-treatment process that increases its hardness without sacrificing its toughness was developed. Different heat-treatment processes were applied to selected die materials including SK4, SKS3, SKD11 and SKH9, which are widely used in the coldforging industry, to obtain sufficient ductility and various values of hardness. The heat-treated die materials were then prepared for specimens used in tensile tests and fatigue tests to obtain their mechanical properties over a wide hardness range. In order to predict the die fatigue life, a theoretical model was also proposed using the die hardness as the major parameter. In conjunction with the finite element simulation results and the mechanical properties of the die materials obtained from the experiments, the theoretical model can predict the die fatigue life to a certain degree.

\section{Heat-treatment processes}

This work examined the fatigue life of common coldforging die materials, such as SK4, SKS3, SKD11 and SKH9, with various values of hardness at sufficient ductility. The chemical composition of each die material is listed in Table 1. The heat-treatment process for the die materials consists of quenching and tempering. For the quenching process, there are three important factors, including: the proper heating temperature up to the range of austenite; the preservation of the transformation of pearlite; and the transformation of martensite or bainite that induces hardening. 
Table 1

The chemical composition of the die materials (wt.\%)

\begin{tabular}{llllllll}
\hline Material & $\mathrm{C}$ & $\mathrm{Mn}$ & $\mathrm{Si}$ & $\mathrm{W}$ & $\mathrm{Cr}$ & $\mathrm{Mo}$ & $\mathrm{V}$ \\
\hline SK4 & $0.90-1.00$ & - & - & - & - & - & - \\
SKS3 & $0.90-1.00$ & $0.90-1.20$ & $<0.35$ & $0.50-1.00$ & $0.50-1.00$ & - & - \\
SKD11 & $1.45-1.65$ & $0.30-0.60$ & $0.20-0.40$ & - & $11.0-13.0$ & $0.70-1.10$ & $0.70-1.00$ \\
SKH9 & $0.70-0.90$ & - & & $6.00-7.00$ & $3.80-4.40$ & $4.00-6.00$ & $1.80-2.30$ \\
\hline
\end{tabular}

After quenching, tempering is usually carried out in order to increase the ductility of the hardened material. In the present study, the four kinds of die material were first quenched from selected temperatures to obtain various values of hardness, then tempering processes were performed to increase the ductility and to release the internal stresses remaining in the quenched die materials. Two tempering cycles were used over different periods for the die materials. In the first tempering process, austenite is mainly transformed into martensite, while the formation of tempered martensite in the second tempering process substantially increases the material ductility and maintains its hardness. The heattreatment conditions for each die material are listed in Table 2.

Fig. 1 shows the relationship between the hardness and the tempering temperature for each die material obtained from the experimental results. It is seen in Fig. 1 that the hardness decreases as higher tempering temperatures are applied to each die material. It is also noted that SK4 and SKS3 behave with a similar trend, and SKD11 and SKH9 possess higher

Table 2

Heat-treatment conditions for the selected die materials

\begin{tabular}{lcllll}
\hline Material & \multicolumn{2}{l}{ Quenching } & & & Tempering \\
\cline { 2 - 3 } \cline { 5 - 6 } & $\begin{array}{l}\text { Temperature } \\
\left({ }^{\circ} \mathrm{C}\right)\end{array}$ & $\begin{array}{l}\text { Time } \\
(\mathrm{min})\end{array}$ & & $\begin{array}{l}\text { Temperature } \\
\left({ }^{\circ} \mathrm{C}\right)\end{array}$ & $\begin{array}{l}\text { Time } \\
(\mathrm{min})\end{array}$ \\
\hline SK4 & 800 & 20 & $150-500$ & 30 \\
SKS3 & 810 & 20 & & $250-500$ & 30 \\
SKD11 & 1010 & 50 & & $150-680$ & 30 \\
SKH9 & 1165 & 3 & $550-720$ & 30 \\
\hline
\end{tabular}

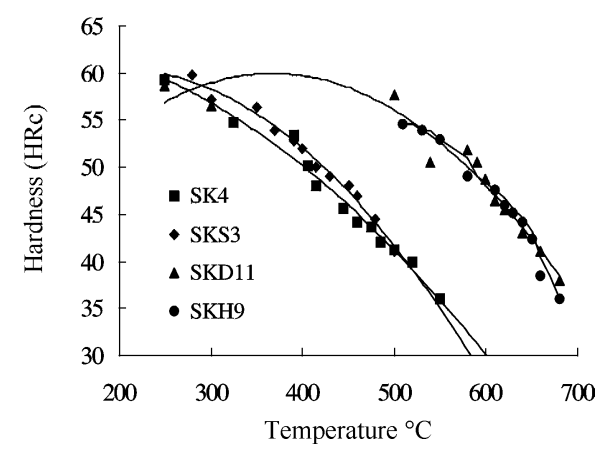

Fig. 1. Relationship between hardness and tempering temperature. values of hardness than those of SK4 and SKS3 when tempered at the same temperature. The values of hardness for each die material obtained in the present study range from HRc 35 to HRc 60.

\section{Mechanical properties}

Since the hardness of the die material is used for the prediction of the fatigue life of the die, the mechanical properties of the die materials are characterized by hardness. In the present study, the die materials with various values of hardness mentioned in the previous section were used for preparing the specimens for tension and impact tests to obtain the relationship between the mechanical properties and the hardness. The mechanical properties of the die materials examined in the present study include Young's modulus, impact strength, elongation, yield stress and fracture strength.

The experimental results show that the Young's modulus of the die material is almost independent of its hardness. The Young's modulus of SK4, SKS3, SKD11 and SKH9 obtained from the experiments for various values of hardness are 198, 202, 208 and $214 \mathrm{GPa}$, respectively. It is to be noted that the Young's modulus differs insignificantly among these four kinds of die materials.

The impact strength of the die material decreases with increasing value of hardness according to the experimental results. In order to retain enough ductility of the hardened die material and prevent the occurrence of brittle fracture, the die materials were heat-treated with additional tempering cycles after being hardened. In the present study, two cycles of tempering processes were conducted to maintain the ductility of the die materials after they had been quenched. The elongation of the die materials also has the same trend as that of impact strength as indicated by the experimental results.

Fig. 2 shows the relationship between the yield stress and the hardness for the four selected die materials as mentioned above. It is seen in Fig. 2 that the yield stress of the die material increases in proportion to the hardness, and the relationship can be formulated by a linear function, as tabulated in Table 3. As for the fracture strength of the die material, this also increases with increasing value of hardness, as shown in Fig. 3. The relationship between the fracture strength and the hardness can be approximately 


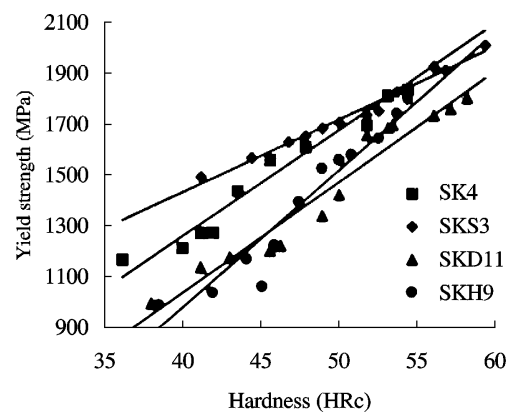

Fig. 2. Relationship between hardness and yield strength.

Table 3

Relationship between hardness $(H)$ and yield strength $(Y)$

\begin{tabular}{ll}
\hline Material & Relationship (MPa) \\
\hline SK4 & $Y=42.1 H-414.5$ \\
SKS3 & $Y=28.7 H+296.1$ \\
SKD11 & $Y=43.8 H-709.9$ \\
SKH9 & $Y=54.2 H-1188.6$ \\
\hline
\end{tabular}

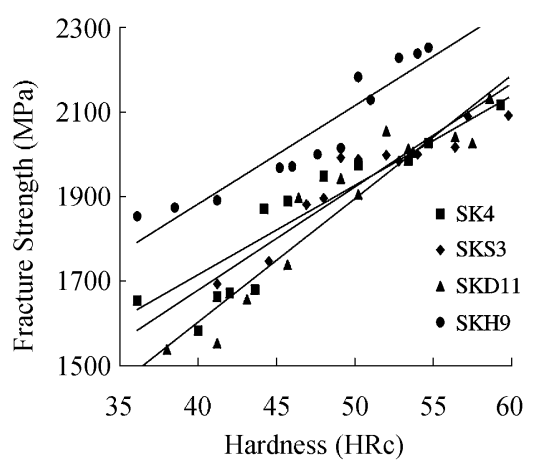

Fig. 3. Relationship between hardness and fracture strength.

expressed by linear equations, the results being given in Table 4.

\section{A theoretical model for the prediction of die fatigue life}

It is well known that the die fatigue life is determined by the loading conditions and the material properties of the die.

Table 4

Relationship between hardness $(H)$ and fracture strength $(F)$

\begin{tabular}{ll}
\hline Material & Relationship (MPa) \\
\hline SK4 & $F=24.4 H+702.9$ \\
SKS3 & $F=21.2 H+869$ \\
SKD11 & $F=29.2 H+436.3$ \\
SKH9 & $F=23.3 H+952.6$ \\
\hline
\end{tabular}

As stated in the previous section, the material properties obtained from the experiments can be related to the hardness of the die. Hence, in the present study, a theoretical model was proposed using the hardness of the die material as the parameter to predict the die fatigue life and to determine the appropriate die hardness in order to obtain an acceptable die service life. Due to the heavy loading applied to the dies used in cold-forging processes, plastic deformation may occur at a position of stress concentration. The die fatigue life drops rapidly if plastic deformation occurs, and, in consequence, the fatigue model requires the consideration of low-cycle fatigue failure.

Under fatigue strain control, the fatigue elastic and plastic strains can be, respectively, expressed as [1-3]:

$\frac{\Delta \varepsilon_{\mathrm{e}}}{2}=\frac{\sigma_{\mathrm{f}}^{\prime}}{E}\left(2 N_{\mathrm{f}}\right)^{B}$

and

$\frac{1}{2} \Delta \varepsilon_{\mathrm{p}}=\varepsilon_{\mathrm{f}}^{\prime}\left(2 N_{\mathrm{f}}\right)^{C}$

where $\Delta \varepsilon_{\mathrm{e}}$ and $\Delta \varepsilon_{\mathrm{p}}$ are the fatigue elastic and plastic strains, respectively, $\sigma_{\mathrm{f}}^{\prime}$ and $\varepsilon_{\mathrm{f}}^{\prime}$ the fatigue strength coefficient and the fatigue ductility coefficient, respectively, $N_{\mathrm{f}}$ the number of fatigue cycles, $B$ a fatigue strength exponent, $C$ a fatigue ductility exponent, and $E$ the Young's modulus. By considering Eqs. (1) and (2), the fatigue life can be expressed as

$\frac{\Delta \varepsilon}{2}=\frac{\Delta \varepsilon_{\mathrm{e}}}{2}+\frac{\Delta \varepsilon_{\mathrm{p}}}{2}=\frac{\sigma_{\mathrm{f}}^{\prime}}{E}\left(2 N_{\mathrm{f}}\right)^{B}+\varepsilon_{\mathrm{f}}^{\prime}\left(2 N_{\mathrm{f}}\right)^{C}$

where $\Delta \varepsilon$ is the fatigue total strain. The occurrence of plastic deformation of die must be avoided by proper die design. The magnitude of the plastic strain at failure is generally much smaller than that of the elastic strain. Therefore, the fatigue total strain is approximately the fatigue elastic strain. However, when the fatigue plastic strain is not small compared to the fatigue elastic strain, the fatigue elastic strain cannot be used directly to represent the total strain. Consequently, the fatigue elastic strain should be corrected by an exponential function in the form of

$\frac{1}{2} \Delta \varepsilon_{\mathrm{e}}=\Delta \varepsilon\left(1-\mathrm{e}^{-A / \Delta \varepsilon}\right)$

where $A$ is a correction factor determined by experiments.

Considering Eqs. (1) and (4):

$\Delta \varepsilon\left(1-\mathrm{e}^{-A / \Delta \varepsilon}\right)=\frac{\sigma_{\mathrm{f}}^{\prime}}{E}\left(2 N_{\mathrm{f}}\right)^{B}$

Eq. (5) represents the approximate relationship between the total fatigue strain and the fatigue life.

For most hardened die materials, the fracture strength $\sigma_{\mathrm{f}}$ is approximately equal to the fatigue strength coefficient $\sigma_{\mathrm{f}}^{\prime}$ $\left(\sigma_{\mathrm{f}}=\sigma_{\mathrm{f}}^{\prime}\right)[1]$. Hence, the fatigue strength coefficient can be replaced by the fracture strength obtained from the tension tests. Since the fracture strength is linearly proportional to the hardness, as stated in the previous section, the fatigue strength coefficient can also be expressed in terms of the die 


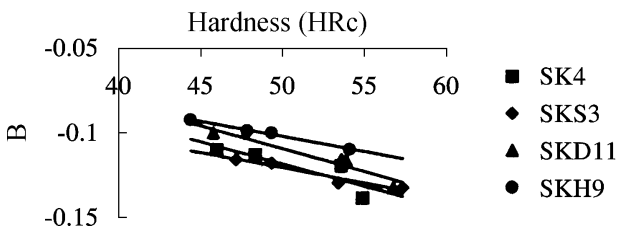

Fig. 4. Relationship between parameter $B$ and hardness.

hardness. In order to simplify the proposed theoretical model, the factor $A$ introduced into Eq. (4), which modifies the fatigue elastic strain into the fatigue total strain, is assumed to be a constant for the die materials investigated. Considering the fatigue test data, $A$ is found to be approximately 0.02 for the die materials investigated in this study. As for the constant $B$ introduced into Eq. (1), this is dependent of the hardness of the die material. For die materials with different values of hardness, $B$ is also different, its value being determined from the fatigue tests. The relationship between $B$ and the material hardness is illustrated in Fig. 4.

With all the material constants determined, the fatigue total strain is yet to be found so that the die fatigue life can be predicted according to Eq. (5). Since the die shape is usually not regular and the loading condition is complex, the deformation of the die is difficult to be found analytically. In the present study, finite element simulations are performed to determine the fatigue strain of the die as it would be of service. The detailed procedure is depicted in the following sections.

\section{Predication of the fatigue life of dies}

In view of Eq. (5), the fatigue total strain can be used to predict the die fatigue life if all other material constants are already determined from the experimental results. In order to facilitate the analysis, finite element simulations were performed in the present study to obtain the fatigue total strain for a selected die set, as shown in Fig. 5. It is seen in Fig. 5 that the die geometry is designed to include a sharp angle at which the plastic deformation induced by the stress concentration is expected to occur, resulting in the low-cycle fatigue failure of the die. All the four die materials examined in the present study were simulated using the same die configuration, shown in Fig. 5, to obtain the fatigue total strain. The material being forged between the top and bottom dies is low carbon steel, and the deformation is assumed to be under plane-strain conditions. The finite element program DEFORM was employed in the present study to perform the simulations.

The simulation processes are illustrated in Fig. 6, and the fatigue total strain was calculated for each die material. Substituting the simulation results into Eq. (5), the predicted fatigue life for each die material was calculated and the service cycles were listed in Table 5 .
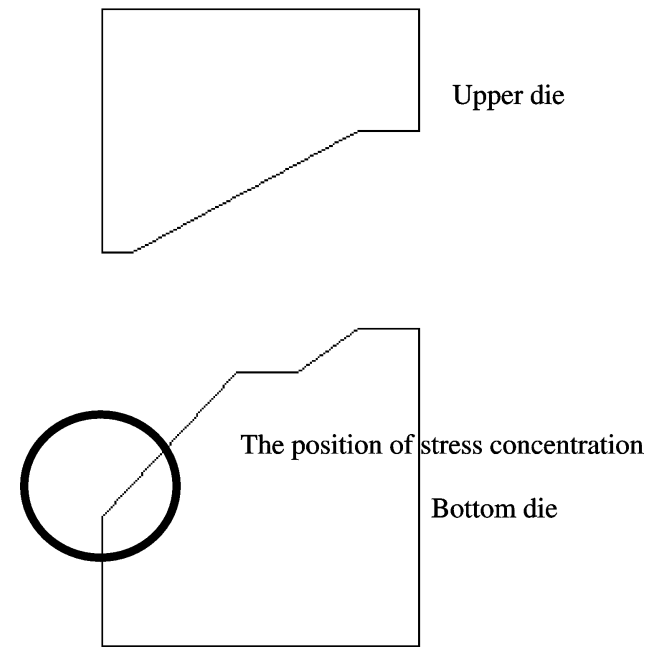

Fig. 5. The selected die configuration for the finite element simulations.

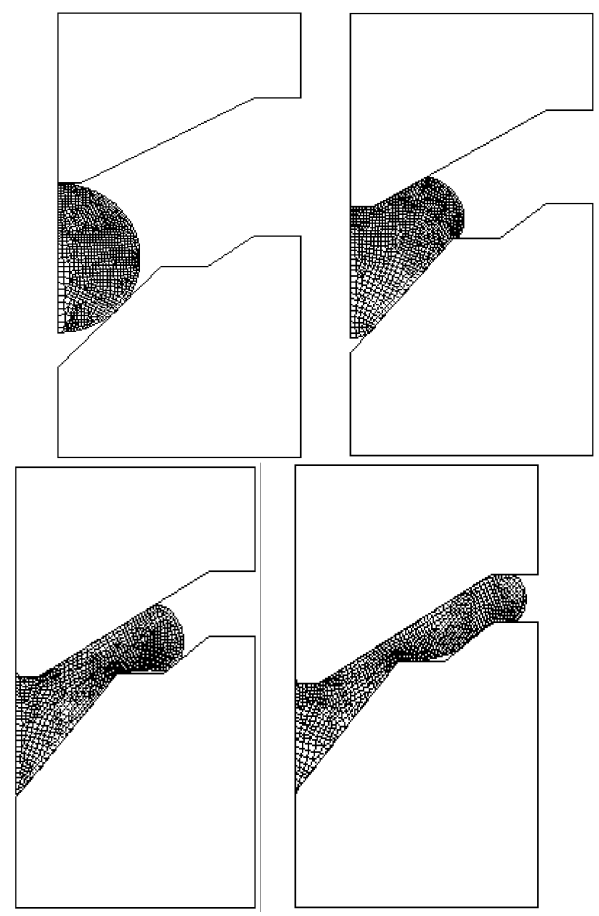

Fig. 6. Finite element simulation of a cold-forging process.

Table 5

Calculated fatigue life of the bottom die

\begin{tabular}{lllll}
\hline $\begin{array}{l}\text { Die } \\
\text { material } \\
(50 \text { HRc })\end{array}$ & $\begin{array}{l}\text { Fatigue } \\
\text { strain } \\
(\text { FEM })\end{array}$ & $\begin{array}{l}\text { Fracture } \\
\text { stress } \\
(\mathrm{MPa})\end{array}$ & $B$ & $\begin{array}{l}\text { Fatigue } \\
\text { life } \\
\text { (cycle) }\end{array}$ \\
\hline SK4 & 0.0098 & 1992.9 & -0.1155 & 843 \\
SKS3 & 0.0115 & 1929 & -0.1206 & 231 \\
SKD11 & 0.0109 & 1896.3 & -0.1091 & 407 \\
SKH9 & 0.01176 & 2117.6 & -0.1019 & 865 \\
\hline
\end{tabular}




\section{Concluding remarks}

The fatigue life of cold-forging dies with various values of hardness were investigated in the present study. Four kinds of die materials, SK4, SKS3, SKD11 and SKH9 were examined. The die materials were heat-treated by the proposed quenching and tempering processes to obtain various values of hardness, while the ductility was retained at a favorable level. The heat-treated die materials were then used to prepare the specimens for the tension and impact tests to construct the relationship between the die material properties and the die hardness.

A simple theoretical model was also proposed using the die hardness and the fatigue total strain as parameters to predict the die fatigue life. With the help of the finite element simulations, the fatigue total strain can be calculated and the die fatigue life is then predictable using the theoretical model. Since the die hardness is easily measurable, the proposed fatigue model is quite efficient in providing a convenient guideline for the estimation of the die quality in any given cold-forging operation. However, the proposed fatigue model is yet to be validated by more experimental data.

\section{Acknowledgements}

The authors wish to thank the National Science Council of the Republic of China for its support under project \#NSC872212-E002-043.

\section{References}

[1] American Society for Testing and Materials, Manual on Low Cycle Fatigue Testing, ASTM STP, Vol. 465, Philadelphia, PA, 1969, pp. 126.

[2] J.A. Bannantin, J.J. Comer, J.L. Handrock, Fundamentals of Metal Fatigue Analysis, Englewood Cliffs, NJ, Prentice-Hall, 1990.

[3] B.I. Sandor, Fundamental of Cyclic Stress and Strain, The University of Wisconsin Press, London, 1972. 